Check for updates

Cite this: RSC Adv., 2019, 9, 2857

Received 24th October 2018

Accepted 2nd January 2019

DOI: $10.1039 / \mathrm{c} 8 \mathrm{ra0} 8818 \mathrm{~g}$

rsc.li/rsc-advances

\section{In situ synthesis of low-cost and large-scale flexible metal nanoparticle-polymer composite films as highly sensitive SERS substrates for surface trace analysis $\uparrow$}

\author{
Chenghua Zong, (D) a Mengyi Ge, ${ }^{a}$ Hong Pan, ${ }^{a}$ Jing Wang, ${ }^{a}$ Xinming Nie, \\ Qingquan Zhang, ${ }^{a}$ Wenfeng Zhao, ${ }^{a}$ Xiaojun Liu ${ }^{\star a}$ and Yang Yu (D)*a
}

\begin{abstract}
Surface-enhanced Raman spectroscopy (SERS) has been one of the most promising analytical tools. Despite many efforts in the design of SERS substrates, it remains a great challenge for creating a general flexible substrate that could in situ detect analytes on diverse objects. Herein, we report our attempt to address this issue by developing a facile and versatile method capable of generating silver/gold nanoparticles in situ on the surface of a cellulose acetate (CA) polymer in a simple, cheap, practical, and capping agent-free way. The as-prepared substrates exhibit excellent sensitivity, which enabled detection of Rhodamine $6 \mathrm{G}$ at concentrations as low as $10^{-12} \mathrm{M}$. Taking advantage of the excellent flexibility and optical transparency of the CA matrix, the highly SERS-active substrate was applied for in situ identification and detection of pesticide residues on fruits. The results indicated that tetramethylthiuram disulfide (TMTD) and thiabendazole (TBZ) can be clearly identified at concentrations as low as $18.05 \mathrm{ng} \mathrm{cm}^{-2}$ and $15.1 \mathrm{ng} \mathrm{cm}^{-2}$, respectively, which were much lower than the maximum permitted residue doses with respect to food safety.
\end{abstract}

\section{Introduction}

Surface-enhanced Raman scattering (SERS) is recognized as one of the most promising sensing techniques due to its ultrasensitive, nondestructive and fingerprint-type way of sensing. ${ }^{1-5}$ It is generally accepted that the strong electromagnetic field enhancement near metallic nanoparticles is mainly responsible for the occurrence of SERS..$^{6-8}$ Due to this, current research about SERS has focused on the design and fabrication of highly sensitive SERS substrates. ${ }^{9-14}$ Metallic colloids were one of the most commonly used substrates mainly due to their ease of preparation. ${ }^{15-17}$ However, the uncontrollable aggregation associated with metallic colloids makes the stability and reproducibility a major challenge in real applications. For this reason, SERS substrates supported by solid matrices are gaining increasing attention. Directly depositing nanostructures on hard templates (e.g. glass, quartz, silicon, etc.) using techniques such as e-beam lithography, colloidal lithography, and vapor deposition has been one of the most commonly used

${ }^{a}$ School of Chemistry and Materials Science, Jiangsu Key Laboratory of Green Synthesis for Functional Materials, Jiangsu Normal University, Xuzhou, Jiangsu, P. R. China, 221116.E-mail:42236117@qq.com; yuyang@jsnu.edu.cn

${ }^{b}$ School of Physics and Electronic Engineering, Jiangsu Normal University, Xuzhou, Jiangsu, 221116, China

$\dagger$ Electronic supplementary information (ESI) available. See DOI: 10.1039/c8ra08818g strategies. ${ }^{18-21}$ Although these substrates offer increased stability and higher tailorability, they suffer from high cost and great deal of skills; especially, they cannot be attached to irregular surfaces due to the lack of flexibility, thereby making in situ surface Raman analysis impossible.

Compared with the traditional rigid substrates, flexible substrates are not only light weighted and portable, but also, most importantly, can be easily wrapped and swabbed onto diverse irregular, nonplanar substrates, which facilitate the detection of toxicants from food surfaces and benefit food safety inspection. With all the advantages offered by flexible SERS, materials such as cotton, paper, textile, and electro-spun fiber have been explored to serve as building blocks. ${ }^{22-26}$ Nevertheless, a nanoparticle concentration and a template-modification step are normally required so as to obtain enough hot spots and to fix the nanoparticles firmly; moreover, the poor optical transparency of the above-mentioned substrates will block the light from direct excitation of SERS on the opposite side, which prevents their applications in direct analysis of molecules adsorbed on the arbitrary substances. To address this dilemma, flexible substrates with transparent polymers (e.g. PE, PET, PDMS, etc.) as scaffolds have been reported. ${ }^{27-34}$ For example, owing to the nanoparticle self-assembly on the water/oil interface and the physical constraint of PMMA and PE, highly active, flexible and transparent substrates have been developed recently. ${ }^{35,36}$ However, the requirement of volatile organic 
solvents is environmentally unfriendly. In many cases, obtaining a perfect self-assembled film is not a facile work, requiring careful control of the experimental parameters. Some other groups have reported the fabrication of SERS substrates based on PDMS and commercial tapes. ${ }^{37-40}$ These flexible and transparent substrates enable new functionalities and applications that were not possible previously, but in these cases, the nanoparticles could be synthesized preliminarily, which faces a high risk of particle aggregation in the following decorating step due to the change in environmental conditions. ${ }^{41-43}$ Moreover, the particles synthesized by the wet-chemistry methods usually contain surfactant ligands on their surface, which may induce noisy SERS signals. To solve this problem, Hasell reported an in situ synthesis approach. Unfortunately, this method requires $24 \mathrm{~h}$ heating under high pressure and most of the formed nanoparticles are embedded inside the polymer film, inducing limited exposure to the analyte. ${ }^{\mathbf{4 4}}$ Recently, Fortuni presented the in situ formation of gold nanostars on the surface of the pre-cured PDMS film. ${ }^{45}$ The main disadvantage of this method is the low nanoparticle covering density, which influences the SERS enhancement effect.

With this in mind, we propose herein a facile strategy, which offered opportunities to circumvent these problems. The strategy depends on the in situ fabrication of $\mathrm{Ag}$ nanoparticles (AgNPs) on a cellulose acetate (CA) polymer film by using hydrazine as the reducing agent and an ethanol/water binary solution as the reaction medium. It was found that the choice of the reaction medium is the key to obtain high-performance SERS films. Several features of such a strategy make it particularly attractive for in situ detection applications: (1) it is simple and convenient, which can be easily scaled up with a low cost; (2) no capping agent is needed, thus avoiding the potential noise signal interference and facilitating their further functionalization for specific detection purposes; (3) thanks to the high flexibility of the CA matrix, the obtained substrate can conform to diverse objects, while its excellent optical transparency ensured efficient passing through of light, which makes in situ and non-intrusive detection of analytes on the arbitrary surface possible. The proof-of-concept experiments demonstrate that the substrates can be used for simultaneous quantification of different pesticide residues on apples from one measurement. Additionally, the proposed strategy can be extended to synthesize other noble metal nanomaterials, thus opening up new avenues for constructing metal nanoparticlepolymer composite films.

\section{Materials and methods}

\subsection{Materials}

All reagents were of analytical grade and used without further purification. Silver nitrate (99\%) was purchased from Alfa Aesar. Gold(III) chloride trihydrate $\left(\mathrm{HAuCl}_{4} \cdot 3 \mathrm{H}_{2} \mathrm{O}\right)$, hydrazine, cellulose acetate, $P$-aminothiophenol ( $p$-ATP, 97\%) and Rhodamine 6G (R6G, 99\%) were purchased from Sigma-Aldrich. Tetramethylthiuram disulfide (TMTD), thiabendazole (TBZ), ethanol and nitric acid were obtained from Aladdin-Reagent Co., Ltd. Deionized water was used throughout the experiment.

\subsection{Instruments}

Scanning electron microscopy (SEM) images were obtained using SU8020 (Hitachi, Japan) at an accelerating voltage of $1 \mathrm{kV}$. SERS spectra were recorded using a portable Raman spectrometer (B\&W Tek Inc., USA) at an excitation wavelength of $785 \mathrm{~nm}$. The spreader (MSK-AFA-ES200) was purchased from Shenzhen Kejing Star Technology GO., LTD.

\subsection{Fabrication of SERS substrates}

Cellulose acetate (CA) powder $(0.6 \mathrm{~g})$ was added into a DMF solution of $\mathrm{AgNO}_{3}(0.05 \mathrm{M}, 4 \mathrm{~mL})$. Then, the mixture was stirred at room temperature for $1 \mathrm{~h}$ until a transparent solution was formed. Subsequently, $0.3 \mathrm{~mL}$ of the resulting solution was coated on a clean glass slide to a final area of $2 \times 3 \mathrm{~cm}$ with a thickness of $300 \mu \mathrm{m}$ using a spreading machine. Then the slide was dried at $80^{\circ} \mathrm{C}$ for $30 \mathrm{~min}$ to promote the solidification of the coated polymer, and during this process, the metal precursor was uniformly doped within the polymer. Finally, the in situ fabrication of $\mathrm{Ag}$ nanoparticles was initiated by immersing the slide into an ethanol/water $(5: 1)$ binary solution containing $4 \mathrm{mM}$ hydrazine for $30 \mathrm{~min}$. The obtained AgNPs@CA composite film was exfoliated from the glass slide. The side of AgNPs layer of AgNPs@CA film exhibits a metallic luster because of electronic coupling of closely packed AgNPs. ${ }^{27}$ The other side of AgNPs@CA film is transparent, and laser can pass through it to reach the AgNPs layer; herein, we define the AgNPs@CA film as transparent SERS substrate. After washing with water for several times, the films were stored in water for further use.

\subsection{SERS measurements}

To test the sensitivity of the as-prepared substrate, R6G was selected as the Raman model probe. In brief, small pieces of the AgNPs@CA composite films were immersed in $2 \mathrm{~mL}$ of aqueous R6G solution of different concentrations for 3 hours, then taken out and dried in air. Afterwards, SERS spectra were obtained from the substrate with a laser power of $40 \mathrm{~mW}$ and an integration time of $2 \mathrm{~s}$. To evaluate the reproducibility of the substrate, the AgNPs@CA composite film was incubated in $2 \mathrm{~mL}$ of the $1 \times 10^{-6} \mathrm{M} p$-ATP ethanol solution for $2 \mathrm{~h}$, followed by rinsing with ethanol and drying in air. Then SERS spectra from 30 randomly selected spots were recorded.

Furthermore, to verify the applicability of the as-prepared film as a SERS substrate for in situ analyte detection, $15 \mu \mathrm{L}$ tetramethylthiuram disulfide (TMTD) and thiabendazole (TBZ) with different concentrations were introduced dropwise onto apple peels $\left(1 \times 1 \mathrm{~cm}^{2}\right)$ using a pipettor. After the solution was dried, the AgNPs@CA composite film pre-stored in water was pasted on the contaminated area and was pressed with a certain pressure for $5 \mathrm{~s}$ to enable close conformal attachment of the film to the contaminated samples. The SERS signals were directly collected from the opposite side of the composite film 5 minutes later. The reproducibility of the SERS signals for in situ 
TMTD and TBZ detection was carried out using a pesticide concentration of $5 \times 10^{-5} \mathrm{M}$. Each pesticide was detected five times, and Raman spectra from 20 randomly selected spots were recorded in each measurement. The corresponding average value was used to represent the result of each measurement.

\section{Results and discussion}

\subsection{Fabrication and optimization of the substrate}

The substrates were successfully synthesized on a large-scale using silver nitrate and cellulose acetate (CA) as starting materials via a facile in situ reduction process. Briefly, CA powder was first mixed with sliver nitrate in DMF solution to form a viscous solution. Then the viscous solution was spread onto a clean glass slide using a spreading machine. Afterwards, the film was dried at $80^{\circ} \mathrm{C}$ for $30 \mathrm{~min}$. During this process, the DMF solution was evaporated and the CA was solidified, affording a transparent film with silver nitrate uniformly embedded inside. Finally, the reduction of silver nitrate was initiated by immersing the film directly into a hydrazine in ethanol/water mixture solution. In this case, ethanol was chosen as the main reaction medium because: (1) hydrazine in ethanol exhibits a lower reduction rate when compared with that in water, thus enabling the formation of nanoparticles in a more controlled way, affording good uniformity and (2) silver nitrate
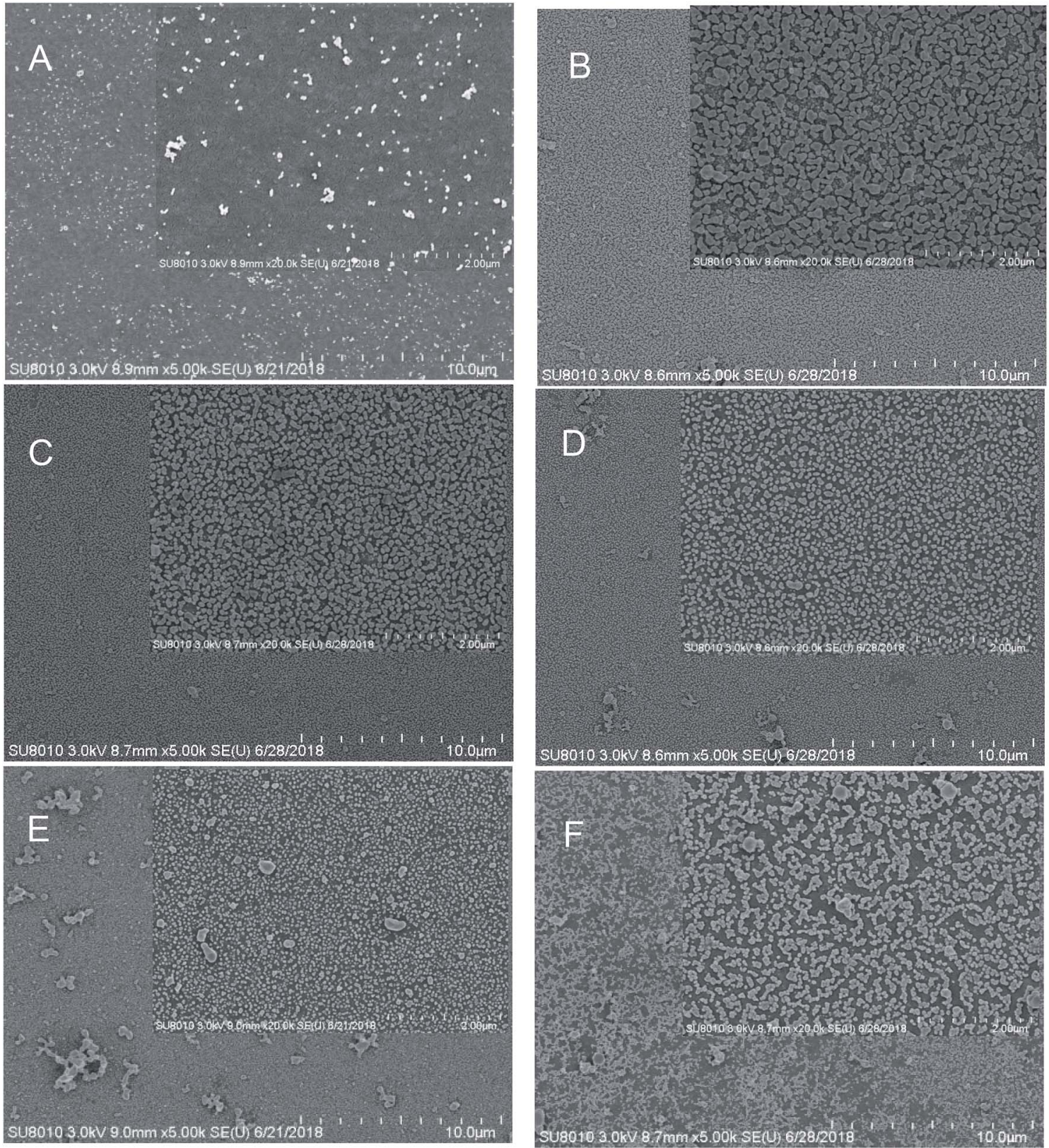

Fig. 1 SEM images of the AgNPs@CA films that were prepared in pure ethanol (A) and in ethanol/water binary solution with a volume ratio of ethanol/water of $7: 1$ (B), $5: 1$ (C), $3: 1$ (D), $2: 1$ (E), and pure water (F). 


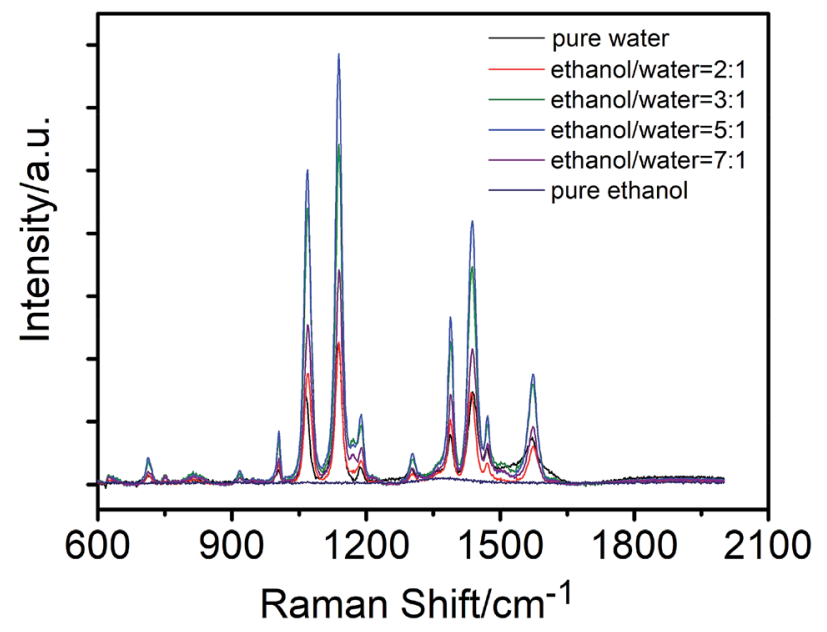

Fig. 2 SERS signals of $1 \times 10^{-6} \mathrm{M} p$-ATP on the AgNPs@CA films prepared at different ethanol/water ratios.

has poor solubility in ethanol, thus preventing them from leaking into the reaction solution. Moreover, it was noted that the presence of little amount of water was essential as in a compared experiment where the film was prepared in ethanol alone, no SERS enhancement was observed. To demonstrate the role of water, the film was prepared at different ethanol/water volume ratios. The SEM results prove that the size and density of the nanoparticles are highly dependent on the initial volume ratio of water in the reaction solution. As shown in Fig. 1A-D, only sparse particles were observed for the film prepared in ethanol alone, but a compact nanoparticle distribution was observed for the film reduced in the ethanol/water binary solution. We suppose that the presence of little amount of water promotes the $\mathrm{Ag}^{+}$inside the film to diffuse to the surface, affording a high surface $\mathrm{Ag}^{+}$concentration and finally a higher particle density. However, further increasing the water content induces a drastic decrease in the uniformity (Fig. 1E and F), which could probably be attributed to the increased $\mathrm{Ag}^{+}$leaking.
The increased turbidity of the reduction medium verified this hypothesis (Fig. S1 $\dagger$ ). The SERS performance of the films prepared at different ethanol/water contents was evaluated. As displayed in Fig. 2, the film that was prepared at a ethanol/water ratio of $5: 1$ possesses the highest SERS enhancement. Based on the above-mentioned result, the volume ratio of ethanol/ water was set as $5: 1$.

Furthermore, the amount of Ag element was also optimized. As displayed in Fig. S2A, $\dagger$ a weak SERS signal of $p$-ATP was initially observed from the film prepared with an $\mathrm{Ag}$ content of $1.25 \mathrm{mg} \mathrm{g}^{-1}$. As the amount of $\mathrm{Ag}$ element gradually increased from 1.25 to $5.0 \mathrm{mg} \mathrm{g}^{-1}$, the Raman signal markedly enhanced. However, any further increase in the amount of Ag element gave rise to a decreased Raman signal. This observation can be understood by comparing the scanning electron microscopy (SEM) images of the samples prepared with different $\mathrm{Ag}$ contents. As shown in Fig. S3A-D, $\dagger$ increasing the gross amount of the $\mathrm{Ag}$ element tends to increase the coverage density of AgNPs. This increased coverage density, which is supposed to deliver a higher density of "hot-spots", is probably responsible for the increased SERS signal intensities. In contrast, the weak SERS signals observed from the samples prepared at higher Ag content are probably caused by the agglomeration phenomenon (Fig. S3A $\dagger$ ). Furthermore, to increase the surface roughness of the AgNPs, the films were treated with nitric acid at different $\mathrm{pH}$ values. As demonstrated in Fig. S2B, $\dagger$ the maximum SERS enhancement was observed at a $\mathrm{pH}$ value of 5 ; nevertheless, further enhancing the acidity lead to a drastic decrease in the SERS signal intensity, which may be attributed to the dissolution of AgNPs under strong acidic conditions.

\subsection{SERS performance of the AgNPs@CA film}

To investigate the SERS sensitivity of the AgNPs@CA film, R6G with different concentrations was adsorbed on the AgNPs@CA film, and their corresponding SERS spectra were obtained as shown in Fig. 3A. As for all the concentrations, the characteristic
A

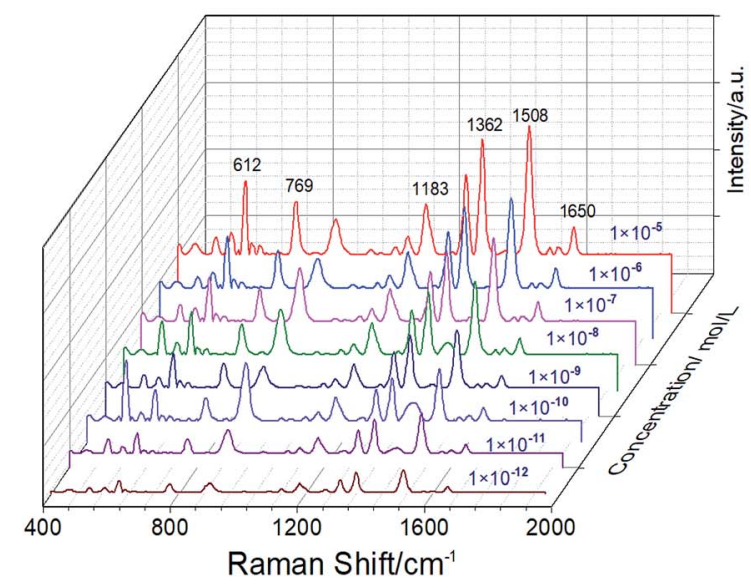

B

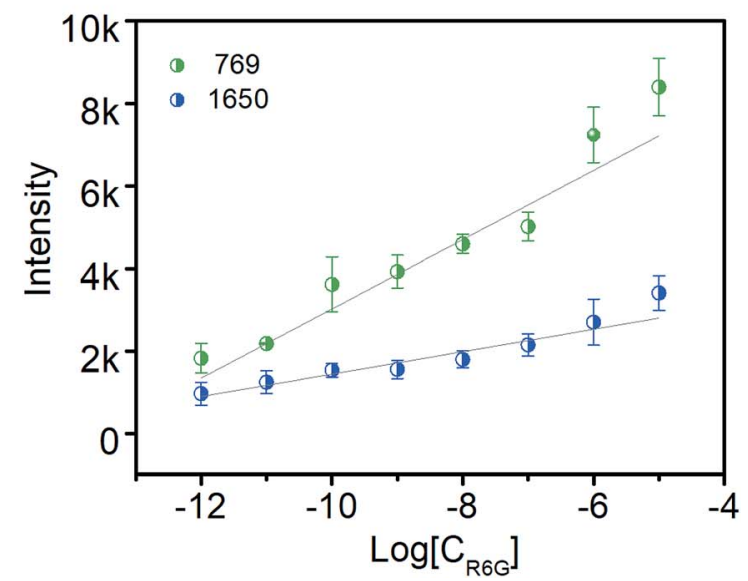

Fig. 3 (A) SERS spectra of R6G with different concentrations. (B) Calibration plot of the SERS intensity at 769 and $1650 \mathrm{~cm}^{-1}$ of R6G introduced dropwise onto the substrates at different concentrations. 
bands of R6G at 612 and $769 \mathrm{~cm}^{-1}$ corresponding to the $\mathrm{C}-\mathrm{C}-\mathrm{C}$ ring in-plane and out-of-plane bending vibrations and the bands at $1183,1362,1508$, and $1650 \mathrm{~cm}^{-1}$ corresponding to the aromatic $\mathrm{C}-\mathrm{C}$ stretching vibrations are observed, ${ }^{29}$ but the intensity and the resolution of the R6G signal declined with the decrease in R6G concentration. When the R6G concentration was reduced to $10^{-12} \mathrm{M}$ on the AgNPs@CA film, Raman signals could be still identified clearly, indicating a high sensitivity of the AgNPs@CA film. Furthermore, the Raman bands at 769 and $1650 \mathrm{~cm}^{-1}$ were employed for the quantitative evaluation of SERS sensitivity. Fig. 3B presents the linear correlation between the peak intensity and the logarithmic concentration of R6G, which indicated a monotonic increase with the logarithmic concentration of R6G from $1 \times 10^{-12}$ to $1 \times 10^{-5}$. To further demonstrate the detection universality, the film was incubated with different concentrations of $p$-ATP. The characteristic modes observed at 1076 and $1581 \mathrm{~cm}^{-1}$ can be assigned to the $\mathrm{C}-\mathrm{S}$ and $\mathrm{C}-\mathrm{C}$ stretching vibrations of $p$-ATP. The other distinct peaks at 1141, 1388 and $1435 \mathrm{~cm}^{-1}$ can be attributed to the fundamental benzene ring vibrations (Fig. S4†). ${ }^{\mathbf{1 6}}$ It is shown that with the decrease in the concentration of $p$-ATP, the intensity of the Raman vibration signals decreases gradually. It should be noted that the vibration modes can still be observed when the concentration of $p$-ATP decreased to $10^{-11}$, indicating the excellent SERS activity of the present AgNPs@CA composite film substrate.

Benefiting from the good optical transparency of the CA matrix ( $~ 93 \%$ at an excitation wavelength of $785 \mathrm{~nm}$ ), the laser can easily penetrate through the CA template to arrive at the assembled AgNPs layer, and consequently motivate the SERS effect. As demonstrated in Fig. 4A and B, the SERS signals collected from both sides (20 points, respectively) of the film were almost identical, and the RSD for the band vibration of $p$ ATP at $1388 \mathrm{~cm}^{-1}$ was $15 \%$, suggesting that the excitation and collection of the Raman signal from the backside are as efficient as from the front side. Notably, this is especially important for noninvasive and direct quantification of contaminant on the object surface. To further analyze the homogeneity and reproducibility, SERS of $1 \times 10^{-6} p$-ATP from 10 different AgNPs@CA composite films were recorded. Fig. 4C and D show the Raman waterfall plots and the corresponding contour plots of 10 different AgNPs@CA composite films. The RSD for the $1388 \mathrm{~cm}^{-1}$ band vibrations of $p$-ATP was $16.3 \%$, demonstrating that the enhancement achieved with the AgNPs@CA film is highly reproducible. In addition, the enhancement factor of the
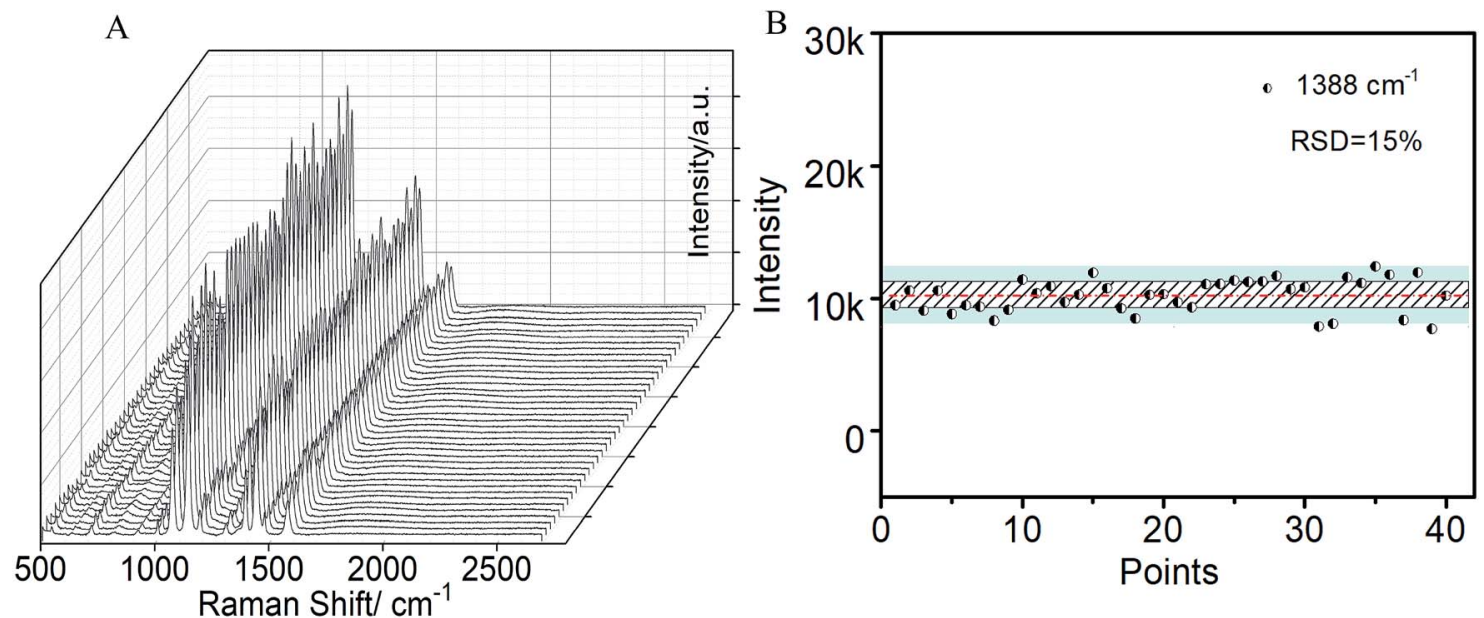

$\mathrm{C}$

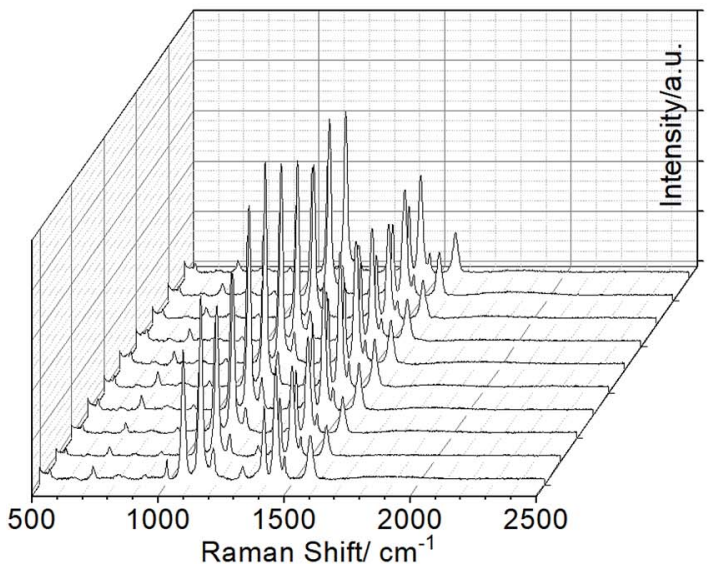

$\mathrm{D}$

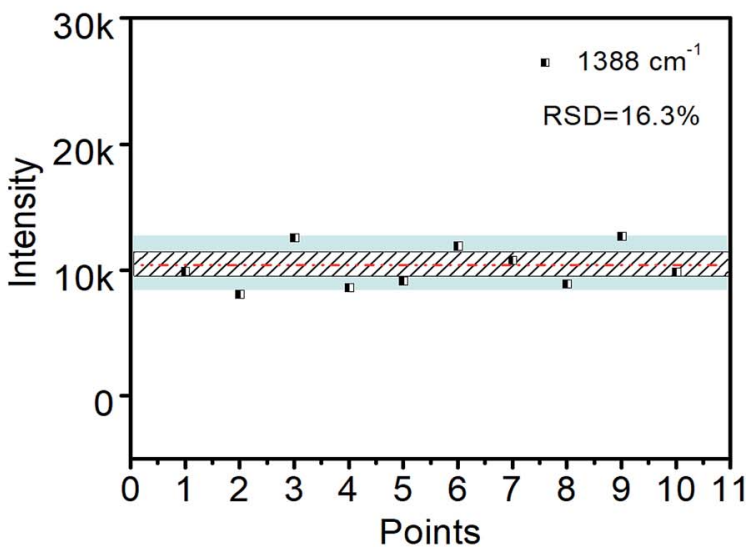

Fig. 4 Reproducibility of the SERS signals on AgNPs@CA films. (A) SERS spectra of $1 \times 10^{-6} p$-ATP recorded from 20 different positions on both side of the AgNPs@CA composite film, respectively. (B) The SERS intensity distribution of the $1388 \mathrm{~cm}^{-1}$ bands. (C) SERS spectra of $p$-ATP ( $\left.1 \times 10^{-6} \mathrm{M}\right)$ recorded from 10 different AgNPs@CA composite films. (D) The corresponding SERS intensity distribution of the $1388 \mathrm{~cm}^{-1}$ bands. The average intensity value is indicated by the red line; intensity variations of $\pm 8 \%$ and $\pm 8-15 \%$ are indicated by the cross grain and light green zones, respectively. 
A

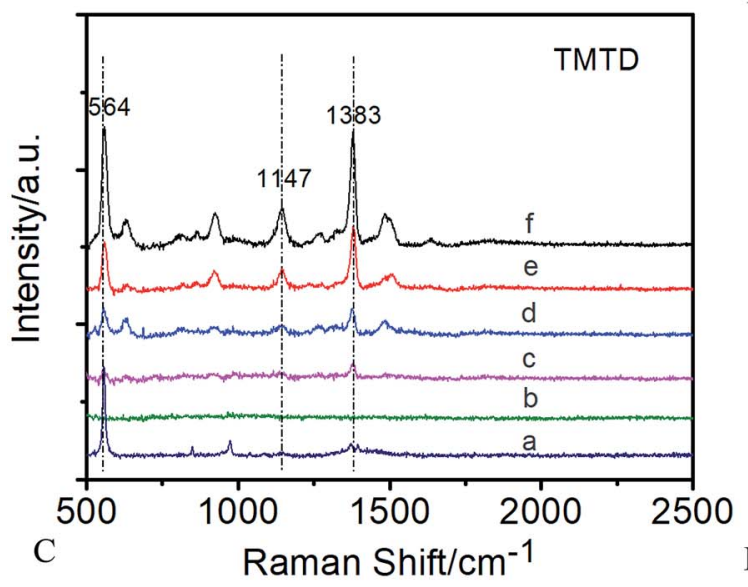

B

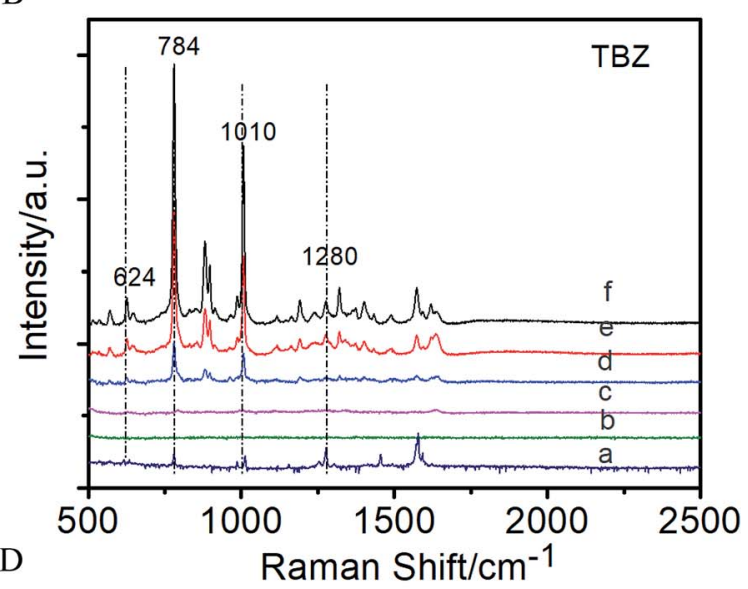

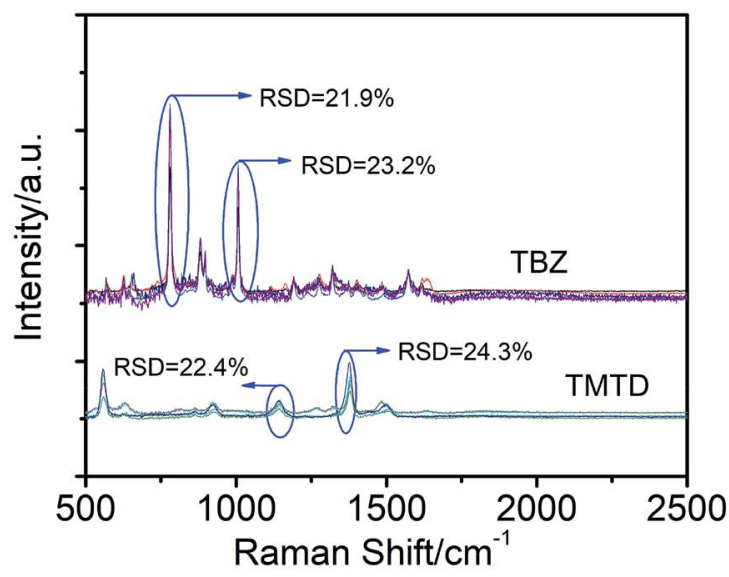

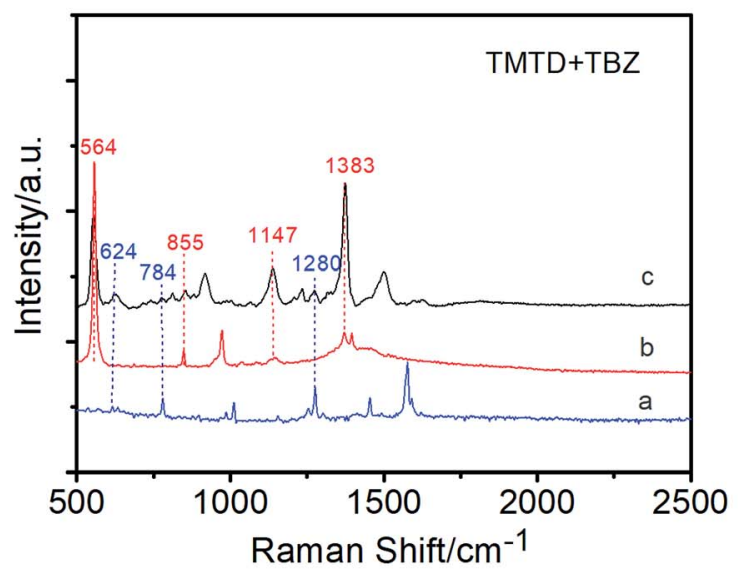

Fig. 5 Raman spectra of (A) TMTD and (B) TBZ: (a) normal Raman band of solid pesticides powder, (b) normal Raman band of 0.1 mM corresponding pesticide on the surface of the apple pieces, (c-f) SERS spectra of different concentrations of TMTD ((c) $18.05 \mathrm{ng} \mathrm{cm}^{-2}$, (d) $36.10 \mathrm{ng}$ $\mathrm{cm}^{-2}$, (e) $180.5 \mathrm{ng} \mathrm{cm}^{-2}$, (f) $360 \mathrm{ng} \mathrm{cm}^{-2}$ ) and TBZ ((c) $15.1 \mathrm{ng} \mathrm{cm}^{-2}$, (d) $30.2 \mathrm{ng} \mathrm{cm}^{-2}$, (e) $151 \mathrm{ng} \mathrm{cm}^{-2}$, (f) $300 \mathrm{ng} \mathrm{cm}^{-2}$ ) obtained directly from the surface of the apple pieces. (C) Reproducibility of the SERS signals for in situ TMTD and TBZ detection (the reproducibility and the RSDs were calculated using an original pesticide concentration of $5 \times 10^{-5} \mathrm{M}$; each pesticide was tested for five times, and 20 points Raman signal were collected each time. The average value of the 20 data is used to represent the results of each measurement). (D) Comparative Raman spectra of the TBZ (a) and TMTD (b) powder, and (c) the mixture of $5 \times 10^{-5}$ M TMTD and TBZ on the apple peel.

AgNPs@CA film was calculated to be $1.8 \times 10^{7}$ by comparing the ratio of the SERS peak intensity of $p$-ATP to its corresponding normal Raman peak intensity [Fig. S5, Text S1†]. These results are remarkable considering the simplicity of preparation, cheapness and robustness of the present AgNPs@CA composite film.

\subsection{Application of the AgNPs@CA film for pesticide detection from fruits}

Pesticide residues pose a great threat to human health, and have aroused worldwide food safety concerns. Although identification of residues can be realized by many methods, one advantage of SERS over them is its capability to perform simultaneous quantification of different components from one measurement because of the unique fingerprint-type way of sensing. To demonstrate the capability of the film for in situ detection of organic pollutants on solid surface, TMTD and TBZ, two kinds of hazardous pesticides that are commonly used in agriculture production were selected. In a proof-of-concept experiment, apple samples were washed with ultrapure water before use, and then cut into small pieces. $15 \mu \mathrm{L}$ pesticide solutions of TMTD and TBZ with different concentrations were spread on the apple peels and dried naturally in air, respectively. Then, the film pre-stored in water was pasted to the surface of the apple pieces and was pressed with a certain pressure for $5 \mathrm{~s}$ to enable close conformal attachment of the film to the contaminated samples. Then the Raman signals were collected directly from the adhered film. As shown in Fig. 5A and B, no evident Raman signals were recorded when a laser was directly shot onto the apple peels spread with $0.1 \mathrm{mM}$ TMTD or TBZ. In contrast, the introduction of the AgNPs@CA film greatly enhanced the Raman signal of the TMTD and TBZ molecules. The detectable concentration for TMTD and TBZ was as low as $18.05 \mathrm{ng} \mathrm{cm}^{-2}$ and $15.1 \mathrm{ng} \mathrm{cm}^{-2}$, respectively, which were much lower than the maximum permitted residue of TMTD and TBZ on apples $\left(\sim 2 \mu \mathrm{g} \mathrm{cm}{ }^{-2}\right.$ for TMTD and $1.2 \mu \mathrm{g} \mathrm{cm}^{-2}$ for TBZ). ${ }^{33,46}$ Furthermore, the 
AgNPs@CA film shows excellent SERS reproducibility for the in situ pesticide inspection, with a RSD value below 0.25 for both TMTD and TBZ (Fig. 5C). Furthermore, to test the possibility for simultaneous detection, $0.05 \mathrm{mM}$ TMTD and TBZ were mixed together and spread on an apple peel. Three main Raman bands of TMTD at 564, 1147 and $1383 \mathrm{~cm}^{-1}$ can be clearly identified, which are attributed to S-S stretching, C-N stretching or $\mathrm{CH}_{3}$ deformation and $\mathrm{C}-\mathrm{N}$ stretching vibrations, respectively. ${ }^{47}$ Compared with the normal Raman result of TBZ (Fig. 5D, pot a), the Raman bands of TBZ collected from apple peel surface can be identified at 624, 784 and $1280 \mathrm{~cm}^{-1}$ (Fig. 5D, pot c), ${ }^{48,49}$ demonstrating great potential of the proposed substrates for simultaneous detection of pesticide residues. Notably, compared with the conventional SERS substrate, which requires pre-extraction of analytes from the complex surfaces, our approach based on free-standing SERS film can fulfill the measurement by just covering the target surface, avoiding tedious sample preparation and contaminating the object.

\subsection{Additional applications}

Generally, hydrazine is a strong reductant, which can be used to synthesize a large number of metal nanoparticles. Therefore, the reported approach can potentially be applicable in synthesis of other metal-based films. To verify this generalizability, a AuNPs@CA film was further prepared with the proposed strategy by using $\mathrm{HAuCl}_{4} \cdot 3 \mathrm{H}_{2} \mathrm{O}$ as the metal precursor. Interestingly, unlike the AgNPs@CA film, it was found that the leakage of $\mathrm{Au}^{3+}$ was considerably apparent in all the studied ethanol/water system, except for the absolute water system, a consequence which we infer to be caused by the imbalance of the diffusion and reduction rates of the $\mathrm{Au}^{3+}$. In the case of an aqueous system, owing to the strong reducibility of the hydrazine, the diffused $\mathrm{Au}^{3+}$ ions can be rapidly reduced, thereby preventing them from leaking into the solution phase. On the contrary, in the ethanol-dominant binary systems, the reducibility of hydrazine weakens, and hence the diffused $\mathrm{Au}^{3+}$ ions (unlike $\mathrm{AgNO}_{3}, \mathrm{HAuCl}_{4} \cdot 3 \mathrm{H}_{2} \mathrm{O}$ are soluble in ethanol) cannot be reduced instantly, leading to its severe leakage. Based on the above-mentioned results, an aqueous solution of hydrazine was used for the preparation of the AuNPs@CA film. Fig. S6 $†$ displays the SEM images of the AuNPs@CA film that was prepared using different $\mathrm{Au}$ contents. With the increase in the $\mathrm{Au}$ content, AuNPs grew larger and the gap distance between AuNPs consequently reduced. When the Au content was higher than $20 \mathrm{mg} \mathrm{g}^{-1}$, the AuNPs tended to pack together. Beside the size change, it was found that the spherical Au nanoparticles transformed to a star shape as the Au content was increased from $2.5 \mathrm{mg} \mathrm{g}^{-1}$ to $10 \mathrm{mg} \mathrm{g}^{-1}$. We believe that these AuNPs@CA composite films may find various applications in the context of novel nanoparticle-based sensing films.

\section{Conclusion}

Herein, we have presented a facile approach for large-scale fabrication of low-cost, flexible and highly active SERS substrates by employing a premixing and then an in situ reduction method. The obtained composite film can conform to the arbitrary surfaces, while the optical transparency allows light interaction with the contact surface, thereby providing the feasibility for in situ and nondestructive detection of analytes on diverse surfaces. Proof-of-concept for in situ detection application of the SERS substrate was demonstrated through direct SERS detection of pesticide residues on apple samples. Moreover, the proposed method has a generalizability that can be extended to the fabrication of other metal-polymer composites, opening up new avenues for developing portable sensors. It is expected that the proposed strategy will bring the SERS technology closer to practical analysis.

\section{Conflicts of interest}

There are no conflicts to declare.

\section{Acknowledgements}

The authors are grateful to the Natural Science Foundation of China (21804060; 21601072), the Jiangsu Province Natural Science Foundation of China (BK20150228), the Natural Science Foundation of the Higher Education Institutions of Jiangsu Province (16KJB510009), Science and Technology Innovation Project of Xuzhou (KC 18137).

\section{References}

1 Y. Wang, B. Yan and L. Chen, Chem. Rev., 2013, 113, 13911428.

2 X. Zou, Y. Wang, W. Liu and L. Chen, Analyst, 2013, 142, 2333-2337.

3 J. Zhang, L. He, P. Chen, C. Tian, J. Wang, B. Liu, C. Jiang and Z. Zhang, Nanoscale, 2017, 9, 1599-1606.

4 C. Jiang, R. Liu, G. Han and Z. Zhang, Chem. Commun., 2013, 49, 6647-6649.

5 Y. Han, S. Liu, B. Liu, C. Jiang and Z. Zhang, RSC Adv., 2014, 4, 2776-2782.

6 H. Hou, P. Wang, J. Zhang, C. P. Li and Y. D. Jin, ACS Appl. Mater. Interfaces, 2015, 7, 18038-18045.

7 V. K. R. a. T. P. Radhakrishnan, ACS Appl. Mater. Interfaces, 2015, 7, 12767-12773.

8 C. Zong, M. Xu, L.-J. Xu, T. Wei, X. Ma, X.-S. Zheng, R. Hu and B. Ren, Chem. Rev., 2018, 118, 4946-4980.

9 P. Xu, X. Han, B. Zhang, Y. Du and H. L. Wang, Chem. Soc. Rev., 2014, 43, 1349.

10 V. Dugandžić, I. J. Hidi, K. Weber, D. Cialla-May and J. Popp, Anal. Chim. Acta, 2016, 946, 73-79.

11 B. Peng, G. Li, D. Li, S. Dodson, Q. Zhang, J. Zhang, Y. H. Lee, H. V. Demir, X. Yi Ling and Q. Xiong, ACS Nano, 2013, 7, 5993-6000.

12 V. K. Rao, P. Ghildiyal and T. P. Radhakrishnan, J. Phys. Chem. C, 2017, 121, 1339-1348.

13 A. Biswas, I. S. Bayer, D. H. Dahanayaka, L. A. Bumm, Z. Li, F. Watanabe, R. Sharma, Y. Xu, A. S. Biris, M. G. Norton and E. Suhir, Nanotechnology, 2009, 20, 325705. 
14 L. Zhang, C. Jiang and Z. Zhang, Nanoscale, 2013, 5, 37733779.

15 S. E. J. Bell and N. M. S. Sirimuthu, Chem. Soc. Rev., 2008, 37, 1012-1024.

16 F. Li, J. Wang, Y. Lai, C. Wu, S. Sun, Y. He and H. Ma, Biosens. Bioelectron., 2013, 39, 82-87.

17 R. M. Jarvis and R. Goodacre, Anal. Chem., 2004, 76, 40-47.

18 H. Im, K. C. Bantz, N. C. Lindquist, C. L. Haynes and S.-H. Oh, Nano Lett., 2010, 10, 2231-2236.

19 A. D. McFarland, M. A. Young, J. A. Dieringer and R. P. Van Duyne, J. Phys. Chem. B, 2005, 109, 11279-11285.

20 C. Han, Y. Yao, W. Wang, L. Qu, L. Bradley, S. Sun and Y. Zhao, Sens. Actuators, B, 2017, 251, 272-279.

21 C. Y. Song, B. Y. Yang, Y. Zhu, Y. J. Yang and L. H. Wang, Biosens. Bioelectron., 2017, 87, 59-65.

22 B. Li, W. Zhang, L. Chen and B. Lin, Electrophoresis, 2013, 34, 2162-2168.

23 C. Wang, B. X. Liu and X. C. Dou, Sens. Actuators, B, 2016, 231, 357-364.

24 Z. W. Wang, G. W. Meng, Z. L. Huang, Z. B. Li and Q. T. Zhou, Nanoscale, 2014, 6, 15280-15285.

25 C. H. Lee, M. E. Hankus, L. Tian, P. M. Pellegrino and S. Singamaneni, Anal. Chem., 2011, 83, 8953-8958.

26 Y. Ke, G. W. Meng, Z. L. Huang and N. N. Zhou, J. Mater. Chem. C, 2017, 5, 1402-1408.

27 H. W. Qiu, M. Q. Wang, S. Z. Jiang, L. Zhang, Z. Yang, L. Li, J. J. Li, M. H. Cao and J. Huang, Sens. Actuators, B, 2017, 249, 439-450.

28 X. Wen, G. Li, J. Zhang, Q. Zhang, B. Peng, L. M. Wong, S. Wang and Q. Xiong, Nanoscale, 2014, 6, 132-139.

29 Z. Zuo, K. Zhu, C. Gu, Y. Wen, G. Cui and J. Qu, Appl. Surf. Sci., 2016, 379, 66-72.

30 S. Emamian, A. Eshkeiti, B. B. Narakathu, S. G. R. Avuthu and M. Z. Atashbar, Sens. Actuators, B, 2015, 217, 129-135.

31 P. Kumar, R. Khosla, M. Soni, D. Deva and S. K. Sharma, Sens. Actuators, B, 2017, 246, 477-486.

32 S. Kumar, P. Goel and J. P. Singh, Sens. Actuators, B, 2017, 241, 577-583.
33 J. L. Jiang, S. M. Zou, L. W. Ma, S. F. Wang, J. S. Liao and Z. J. Zhang, ACS Appl. Mater. Interfaces, 2018, 10, 9129-9135.

34 S. Liu, C. Jiang, B. Yang, Z. Zhang and M. Han, RSC Adv., 2014, 4, 42358-42363.

35 L. B. Zhong, J. Yin, Y. M. Zheng, Q. Liu, X. X. Cheng and F. H. Luo, Anal. Chem., 2014, 86, 6262-6267.

36 N. N. Zhou, G. W. Meng, Z. L. Huang, Y. Ke, Q. T. Zhou and X. Y. Hu, Analyst, 2016, 141, 5864-5869.

37 J. M. Chen, Y. J. Huang, P. Kannan, L. Zhang, Z. Y. Lin, J. W. Zhang, T. Chen and L. H. Guo, Anal. Chem., 2016, 88, 2149-2155.

38 Z. Liu, L. Cheng, L. Zhang, C. Jing, X. Shi, Z. Yang, Y. Long and J. Fang, Nanoscale, 2014, 6, 2567-2572.

39 A. Shiohara, J. Langer, L. Polavarapu and L. M. Liz-Marzan, Nanoscale, 2014, 6, 9817-9823.

40 V. Suresh and F. L. Yap, RSC Adv., 2015, 5, 61671-61677.

41 D. Vilela, M. C. González and A. Escarpa, Anal. Chim. Acta, 2012, 751, 24-43.

42 K. Saha, S. S. Agastic, C. Kim, X. Li and V. M. Rotello, Chem. Rev., 2012, 112, 2739-2779.

43 X. Liu, Z. Wu, Q. Zhang, W. Zhao, C. Zong and H. Gai, Anal. Chem., 2016, 88, 2119-2124.

44 T. Hasell, L. Lagonigro, A. C. Peacock, S. Yoda, P. D. Brown, P. J. A. Sazio and S. M. Howdle, Adv. Funct. Mater., 2008, 18, 1265-1271.

45 B. Fortuni, Y. Fujita, M. Ricci, T. Inose, R. Aubert, G. Lu, J. A. Hutchison, J. Hofkens, L. Latterini and H. Uji-i, Chem. Commun., 2017, 53, 5121-5124.

46 H. Cui, S. Y. Li, S. Z. Deng, H. J. Chen and C. X. Wang, ACS Sens., 2017, 2, 386-393.

47 B. Liu, G. Han, Z. Zhang, R. Liu, C. Jiang, S. Wang and M. Y. Han, Anal. Chem., 2012, 84, 255-261.

48 J. Jiang, S. Zou, L. Ma, S. Wang, J. Liao and Z. Zhang, ACS Appl. Mater. Interfaces, 2018, 10, 9129-9135.

49 J. Feng, Y. Hu, E. Grant and X. Lu, Food Chem., 2018, 239, 816-822. 